\title{
Publication misrepresentation among neurosurgery residency applicants: an increasing problem
}

\author{
Heather M. Kistka, MD, ${ }^{1}$ Arash Nayeri, BS, ${ }^{2}$ Li Wang, MS, ${ }^{3}$ Jamie Dow, EdM, ${ }^{1}$ \\ Rameela Chandrasekhar, PhD, ${ }^{3}$ and Lola B. Chambless, MD ${ }^{1}$ \\ ${ }^{1}$ Department of Neurosurgery, Vanderbilt University Medical Center; ${ }^{2}$ Vanderbilt University School of Medicine; and ${ }^{3}$ Department \\ of Biostatistics, Vanderbilt University School of Medicine, Nashville, Tennessee
}

\begin{abstract}
OBJECTIVE Misrepresentation of scholarly achievements is a recognized phenomenon, well documented in numerous fields, yet the accuracy of reporting remains dependent on the honor principle. Therefore, honest self-reporting is of paramount importance to maintain scientific integrity in neurosurgery. The authors had observed a trend toward increasing numbers of publications among applicants for neurosurgery residency at Vanderbilt University and undertook this study to determine whether this change was a result of increased academic productivity, inflated reporting, or both. They also aimed to identify application variables associated with inaccurate citations.
\end{abstract}

METHODS The authors retrospectively reviewed the residency applications submitted to their neurosurgery department in $2006(n=148)$ and $2012(n=194)$. The applications from 2006 were made via SF Match and those from 2012 were made using the Electronic Residency Application Service. Publications reported as "accepted" or "in press" were verified via online search of Google Scholar, PubMed, journal websites, and direct journal contact. Works were considered misrepresented if they did not exist, incorrectly listed the applicant as first author, or were incorrectly listed as peer reviewed or published in a printed journal rather than an online only or non-peer-reviewed publication. Demographic data were collected, including applicant sex, medical school ranking and country, advanced degrees, Alpha Omega Alpha membership, and USMLE Step 1 score. Zero-inflated negative binomial regression was used to identify predictors of misrepresentation.

RESULTS Using univariate analysis, between 2006 and 2012 the percentage of applicants reporting published works increased significantly (47\% vs $97 \%, p<0.001)$. However, the percentage of applicants with misrepresentations $(33 \%$ vs $45 \%$ ) also increased. In 2012, applicants with a greater total of reported works ( $<<0.001)$ and applicants from unranked US medical schools (those not ranked by US News \& World Report) were more likely to have erroneous citations ( $p=$ 0.038).

CONCLUSIONS The incidence of legitimate and misrepresented scholarly works reported by applicants to the authors neurosurgery residency program increased during the past 6 years. Misrepresentation is more common in applicants from unranked US medical schools and those with a greater number of reported works on their application. This trend is concerning in a profession where trustworthiness is vital. To preserve integrity in the field, programs should consider verifying citations prior to submitting their rank lists.

http://thejns.org/doi/abs/10.3171/2014.12.JNS141990

KEY WORDS residency; publications; misrepresentation; scientific integrity; honor principle

$\mathrm{M}$ ISREPRESENTATION of professional achievements is a well-recognized phenomenon across a variety of professions. Scholarly works in medicine are particularly vulnerable to this type of embellishment. ${ }^{6,9,13}$ The prevalence of scholarly misrepresentation has been studied in applicants for residency and fellowship in sev- eral medical and surgical subspecialties, ${ }^{1-5,7,10-12,14,15,18-23}$ and published rates vary widely from $1.8 \%$ to $30.2 \% .^{11,21}$ The most recent report in neurosurgery, by Cohen-Gadol et al., ${ }^{4}$ demonstrated below-average rates of misrepresentation for neurosurgery applicants from 2001-2002 compared with other fields, leading to the conclusion that the

ABBREVIATIONS AOA = Alpha Omega Alpha; ERAS = Electronic Residency Application Service; SF Match = San Francisco Match; USMLE = United States Medical Licensing Examination. ACCOMPANYING EDITORIAL See pp 190-192. DOI: 10.3171/2015.1.JNS142803. SUBMITTED August 27, 2014. ACCEPTED December 9, 2014. INCLUDE WHEN CITING Published online July 24, 2015; DOI: 10.3171/2014.12.JNS141990. DISCLOSURE Institutional research funding was used for statistical analysis and support. 
majority of applicants to residency programs in neurological surgery are honest in their reporting.

In recent years, we have noticed a dramatic increase in the number of publications reported by applicants to the neurosurgery residency program at our institution. Our initial assumption was that this was due to increasing competitiveness in the applicant pool leading to increased pressure on applicants to have substantial publications before applying to a neurosurgery residency program. However, we also thought it prudent to determine whether this same pressure led to dishonest embellishments of academic achievements as well. Furthermore, in an effort to streamline the application surveillance process, we sought to identify other application variables that may serve as "red flags" for further scrutiny of citations.

\section{Methods}

After obtaining exemption from our institutional review board, we retrospectively reviewed all 2006 SF Match (San Francisco Match) applications $(\mathrm{n}=148)$ and 2012 ERAS (Electronic Residency Application Service) applications $(n=194)$ submitted by candidates for our neurosurgical residency program. Data collected included sex, scholarly work, medical school, advanced degrees (MBA, MPH, JD, PhD, etc.), Alpha Omega Alpha (AOA) membership, and United States Medical Licensing Examination (USMLE) Step 1 score. US News \& World Report research ranking for the year of application was recorded for US medical schools. Ninety-one schools were ranked in 2007 and 89 in 2013. Cited works were included if they were reported as published, accepted, in press, or without a specific designation and listed in the "Peer Reviewed Journal Article/Abstracts," "Peer Reviewed Journal Article/Abstracts (other than published)," and "Peer Reviewed Online Publication" sections of the ERAS application.

Oral presentations, posters, chapters, and articles listed as submitted, provisionally accepted, non-peer reviewed, or other were excluded from analysis. The accuracy of all reported published work was verified via online search of Google Scholar (scholar.google.com), PubMed (http:// www.ncbi.nlm.nih.gov/pubmed), and journal websites. If a work could not be verified via online search, the journal was contacted directly via phone and/or e-mail. Publications that were erroneously reported or were unverifiable through direct contact with the publication offices were considered misrepresentations. The definition of erroneous reporting included reporting work that did not exist, incorrectly listing the applicant as first author, incorrectly listing a work as peer reviewed, or listing an online-only publication in the "Peer Reviewed Journal Article/Abstracts" section of the application rather than the "Peer Reviewed Online Publication" section. If a journal could not be reached for verification, the work was excluded from the analysis. Retrieval rate was calculated as the total number of papers and abstracts in journals that could be reached for comment divided by the total number of reported publications. Minor inaccuracies in citations, such as incorrect page numbers, were not considered misrepresentation. De-identified information was entered into a Microsoft Excel spreadsheet.
The primary endpoints included misrepresentation of abstracts, misrepresentation of papers, and misrepresentation of the total number of published works (abstracts and papers combined). The covariates included in multivariate models were: sex, AOA member, advanced degree, USMLE Step 1 score, US News \& World Report research ranking, foreign medical school graduate, and the total number of reported works (abstracts, papers, posters, books, etc.). Descriptive statistics for continuous and categorical variables were presented as median (with interquartile range [IQR]) and frequency (percentage), respectively. Comparison of continuous baseline demographics between the 2 application years was conducted using the Wilcoxon rank-sum test, and the Pearson chisquare test was used to compare categorical variables between groups. Zero-inflated negative binomial regression was used to evaluate misrepresentation among applicants who reported published work. All model assumptions were evaluated. All tests were 2-tailed, and statistical significance was set a priori at 5\%. Statistical analyses were performed using open source $\mathrm{R}$ statistical software (version 3.0.2).

\section{Results \\ Demographic Characteristics}

Vanderbilt University Medical Center received 148 applications for neurosurgical residency in 2006 and 194 in 2012. The overall demographic characteristics of the applicants were similar between application years. Males accounted for $72 \%$ of candidates in 2006 and $66 \%$ in 2012 (p $=0.27$ ). Similar percentages of applicants were members of AOA (13\% vs $19 \%, \mathrm{p}=0.15)$ and graduates of foreign medical schools $(20 \%$ vs $19 \%, \mathrm{p}=0.81)$ in the 2 application years. The median USMLE Step 1 score $(230$ vs $243, \mathrm{p}<0.001)$ and the percentage of applicants who had advanced degrees (19\% vs 34\%, $p=0.002)$ were significantly higher in 2012 (Table 1).

\section{Verified Publications}

A total of 73 abstracts and 169 articles met inclusion criteria for 2006; these figures increased to 211 abstracts and 687 articles for 2012. In 2012, significantly more applicants reported published works $(97 \%$ vs $47 \%$, p < 0.001 ), and there was a statistically significant increase in the median number of published works per applicant (3 vs $0, p<0.001)$ (Table 1). The overall retrieval rate was similar for the 2 years studied (96\% in 2006 and $98 \%$ in $2012, p=0.104)$.

\section{Misrepresentation}

In 2006, 23 (33\%) of the 69 applicants who reported a published abstract or paper had at least 1 erroneous citation. This rate increased to 84 (45\%) of 188 in 2012. In addition, more applicants had multiple inaccuracies in 2012 (Fig. 1). The most common form of misrepresentation in 2012 was improperly listing an online only publication in the section for peer-reviewed, published journals $(n=$ 125) rather than the section for online publications. This was followed by articles and abstracts that did not exist ( $\mathrm{n}=15$ in 2006, $\mathrm{n}=57$ in 2012) and abstracts that were 
TABLE 1. Demographic characteristics and publication record of applicants in 2006 and 2012

\begin{tabular}{|c|c|c|c|}
\hline Variable & $2006(n=148)$ & $2012(n=194)$ & p Value \\
\hline \multicolumn{4}{|l|}{ Sex, n (\%) } \\
\hline Male & $106(72 \%)$ & $128(66 \%)$ & $0.27^{*}$ \\
\hline Female & $42(28 \%)$ & $66(34 \%)$ & \\
\hline USMLE Step 1 score, median (IQR) & $230(214-244)$ & $243(228-253)$ & $<0.001 \dagger$ \\
\hline AOA membership, $\mathrm{n}(\%)$ & $19(13 \%)$ & $36(19 \%)$ & $0.15^{*}$ \\
\hline Other advanced degree, $\mathrm{n}(\%)$ & $28(19 \%)$ & $66(34 \%)$ & $0.002^{*}$ \\
\hline Foreign medical school graduate, $\mathrm{n}(\%)$ & $29(20 \%)$ & $36(19 \%)$ & $0.81^{*}$ \\
\hline Ranked US medical school, $\mathrm{n}(\%)$ & $90(61 \%)$ & $126(65 \%)$ & $0.43^{*}$ \\
\hline Total no. of reported works $\ddagger$ per applicant, median (IQR) & $2.5(0.0-6.00)$ & $9.00(6.00-17.00)$ & $<0.001 \dagger$ \\
\hline No. of applicants w/ peer-reviewed publications (\%) & $69(47 \%)$ & $188(97 \%)$ & $<0.001^{*}$ \\
\hline Abstracts & $42(28 \%)$ & $97(50 \%)$ & $<0.001^{*}$ \\
\hline Articles & $61(41 \%)$ & $177(91 \%)$ & $<0.001^{*}$ \\
\hline No. of peer-reviewed publications per applicant, median (IQR) & $0.00(0.00-2.00)$ & $3.00(1.00-6.00)$ & $<0.001 \dagger$ \\
\hline Abstracts & $0.00(0.00-1.00)$ & $0.50(0.00-1.00)$ & $<0.001 \dagger$ \\
\hline Articles & $0.00(0.00-1.20)$ & $2.00(1.00-5.00)$ & $<0.001 \dagger$ \\
\hline Abstract \& paper retrieval rate & $231 / 242(96 \%)$ & $877 / 898(98 \%)$ & $0.104^{*}$ \\
\hline
\end{tabular}

presented at meetings only but were erroneously listed as peer-reviewed publications $(n=18$ in $2006, n=42$ in 2012) (Fig. 2).

Multivariate, zero-inflated negative binomial regression analysis of misrepresentation in 2012 revealed that for every unit increase in total reported works, the expected number of misrepresentations increased by $4 \%$ if all other variables in the model were held constant $(\mathrm{p}<0.001)$. Additionally, a graduate of an unranked US medical school is expected to have $84 \%$ more misrepresentations compared with other applicants with otherwise similar credentials
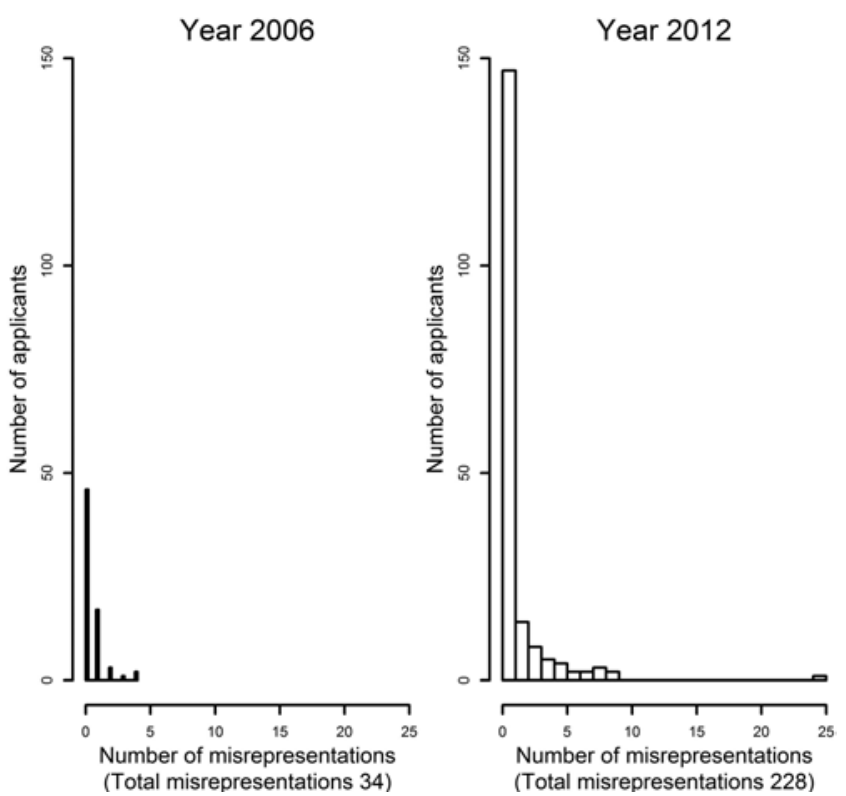

FIG. 1. Histogram illustrating the number of misrepresentations per applicant in 2006 and 2012 $(\mathrm{p}=0.038)$. There was also a trend for applicants who were AOA members $(p=0.079)$ to have fewer inaccuracies. There is no evidence that sex, advanced degrees, or USMLE Step 1 score were associated with the accuracy of citations (Table 2, Fig. 3).

\section{Discussion}

Reports of publication misrepresentation among applicants for residency positions in surgical subspecialties, including neurosurgery, have traditionally reported a low rate of errors compared with other fields. , $^{1,5,10,11,14,19,21-23}$ Disparate results abound in the literature on this topiclikely due to the thoroughness of search criteria, definition of misrepresentation, and variability between applicant populations. ${ }^{11,22}$ However, one must also consider that the landscape of the application process and pool of applicants evolves over time. Our results indicate that the proportion of erroneous reporting has increased in the past 6 years. These changes could reflect a difficulty with the application or, more disturbingly, a decline in academic integrity. Regardless of the cause, the issue of inaccurate citations needs to be addressed to ensure a fair and equal application process for all residency candidates. The data highlight ways in which the application process and ERAS may be improved to avoid unintentional errors as well as provide insight into particular situations in which programs may consider performing additional surveillance.

The major strength of this study is the examination of multiple time points. Studies in various subspecialties have reported their current rates of misrepresentation. However, those are static analyses, and comparisons with historical data are difficult due to the aforementioned variability in study design. This study evaluated the data from 2 time points, using identical search criteria and statistical 
Type of misrepresentation - 2006 $n=34$

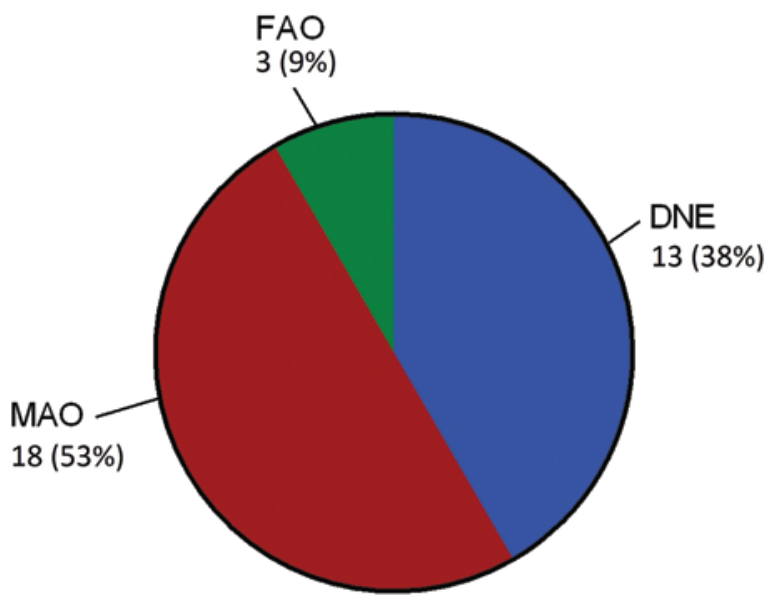

Type of misrepresentation - 2012 $n=228$



FIG. 2. Pie charts illustrating the relative prevalence of each type of misrepresentation in 2006 and 2012. DNE = does not exist (reporting work that does not exist); FAO = false author order (incorrectly listing the applicant as first author); MAO = meeting abstract only (abstract presented at meeting); NPR = not peer reviewed (incorrectly listing a work as peer reviewed); OPO = online publication only (listing an online-only publication in the "Peer Reviewed Journal Article/Abstracts" section of the application rather than the "Peer Reviewed Online Publication" section). Figure is available in color online only.

analysis, allowing for more accurate comparisons between recent data and historical norms. Although changes in the application process make direct statistical comparison difficult, this method provides assurance that the differences seen are due to changes in the applicant pool or application process rather than being due to a change in methodology.

Another 2-time-point analysis was performed by Konstantakos et al. in 1999 and 2005 and 2006. ${ }^{5,14}$ These authors reported a $2 \%$ increase in erroneous reporting in applicants in orthopedic surgery from $18 \%$ to $20.6 \%$. The modest size of this increase compared with our finding could be due to a number of factors. First, it is possible that the substantial increase in erroneous reporting is a new phenomenon over the past 6 years studied in our sample rather than the prior 8 years studied by Konstantakos et al. Second, our results are each from 2 single years at a single institution, whereas the previous report compared data from 2 years at 1 institution to data from 1 year at a different institution.

An additional distinction of our study is that we sepa-

TABLE 2. Effect of applicant characteristics on 2012 rates of misrepresentation

\begin{tabular}{lccc}
\hline \multicolumn{1}{c}{ Variable } & $\begin{array}{c}\text { Exp } \\
\text { (coefficient) }^{*}\end{array}$ & $95 \% \mathrm{Cl}$ & p Value \\
\hline USMLE Step 1 score & 1.00 & $0.99-1.02$ & 0.656 \\
\hline Total no. of reported works & 1.04 & $1.03-1.06$ & $<0.0001$ \\
\hline AOA member & 0.56 & $0.29-1.07$ & 0.079 \\
\hline Advanced degree & 0.99 & $0.60-1.61$ & 0.953 \\
\hline Male sex & 1.27 & $0.79-2.05$ & 0.321 \\
\hline Unranked US medical school & 1.84 & $1.03-3.26$ & 0.038 \\
\hline
\end{tabular}

* Zero-inflated negative binomial regression. Exponential of coefficient $>1$ conveys higher than expected counts of misrepresentation. rate published articles from abstracts. These are significantly different types of publications which require vastly dissimilar time commitments, experience, and scientific rigor. They should be weighted accordingly when reviewing an application. However, the current organization of the ERAS application includes peer-reviewed articles and abstracts under the same heading with no way to quickly distinguish between the two, which can be misleading to

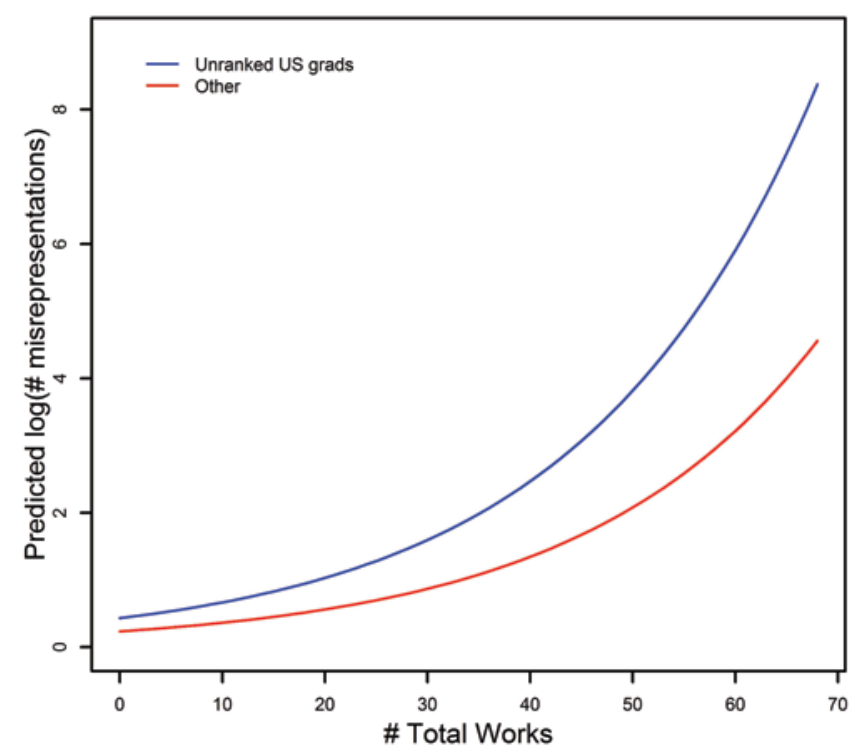

FIG. 3. Graphical depiction of the prevalence of misrepresentation according to number of total works and medical school ranking. Adjusted to male applicant with advanced degree and 2 publications, USMLE Step 1 score of 238, and AOA member. Unranked US grads = graduates of unranked US medical schools (not ranked by US News \& World Report). Figure is available in color online only. 
faculty members reviewing the application and confusing to applicants seeking to accurately cite their work.

Given the fairly high rate of misrepresentation demonstrated by our study and the recent dramatic increase in erroneous reporting, we are anxious to decipher the motivation underlying this behavior. Previous authors ${ }^{14,21}$ have described potential reasons for erroneous reporting. First there is a widespread belief that a greater number of academic works gives applicants an advantage in the application process. ${ }^{16}$ Indeed, a recent survey of Canadian neurosurgeons reported that $78 \%$ felt pressure to publish and that the desire to obtain a good job was a significant research motive. ${ }^{8}$ Furthermore, all medical students at our institution who are interested in neurosurgery are urged to become involved in research projects both for their education and to increase their chances of matching at the program of their choice. Since there continue to be more applicants than residency positions in neurosurgery (314 applicants for 204 positions in the 2013 Match) ${ }_{17}^{17}$ the desire to artificially augment publication records in order to be viewed more favorably by potential programs may be a factor.

Furthermore, there may be a perception among applicants that embellishing a curriculum vitae is common practice, and they may therefore believe that they are at a distinct disadvantage if they do not do so themselves. Additionally, some applicants may simply believe that the benefit of matching at the program of their choice outweighs the risk of a falsification being discovered. It has been suggested that relatively lucrative procedural subspecialties, such as neurosurgery, are more vulnerable to a subpopulation willing to take increased risk to achieve increased financial rewards. ${ }^{21}$ Finally, the misrepresentation of work might simply be a mistake.

Unfortunately, there is no way to discern which contributing factors are the most salient. Although we believe in giving applicants the benefit of the doubt, one would not expect the frequency of unintentional oversights to increase substantially over time. Furthermore, it is difficult to imagine that well-educated applicants would make a large number of errors on a document critical to their future success; nor is the tendency to make mistakes on vital documents a trait of an ideal neurosurgery residency candidate. Therefore, we feel it is doubtful that clerical errors made a substantial contribution to these results.

The increase in misrepresentations could partially be explained by the change in application format from SF Match to ERAS that occurred between 2007 and 2008. Whereas the SF Match applications required the applicants to independently define their work, the 2012 ERAS application (like the current version available at https://www. aamc.org/students/download/424186/data/worksheet 2016 . pdf) provided 9 categories (peer reviewed journal articles/ abstracts, peer reviewed articles/abstracts other than published, peer reviewed book chapter, scientific monograph, other articles, poster presentation, oral presentation, peer reviewed online publication, and non-peer-reviewed online publication). The presumed intent of categories is to remove any ambiguity that may result from freehand self-report, a concern raised by previous authors on this subject. ${ }^{14}$ However, our review found far fewer cases of misleading reporting in the freehand applications.
It is conceivable that the existence of well-defined categories opens the door for unintended misclassification of works by applicants who may not be familiar with the terminology. Investigators who have questioned applicants regarding misrepresentations have found that some did not understand that their works were not peer reviewed. ${ }^{1,15} \mathrm{Al}$ though this may be true in select situations, the finding of more misrepresentations from applicants with large numbers of publications contradicts this theory. An applicant with a prolific publication record is presumably immersed in an academic environment and should have an adequate knowledge of the terminology and access to mentors to avoid such errors. An alternative possibility is that multiple categories create ways for information to be misrepresented in a way that may not seem like a flagrant offense. Listing a non-peer-reviewed article or online-only publication under the peer-reviewed heading, although dishonest, may seem like a "white lie" that is unlikely to be caught.

There are several limitations to our study. It is retrospective and is therefore susceptible to bias. We narrowly defined misrepresentation to include confirmation through direct journal contact so as not to overestimate the results. Our exhaustive search accounted for misspellings and clerical errors. Even so, there is a possibility that a legitimate work with a grossly inaccurate citation was labeled a misrepresentation. Also, the pool of applicants and the application used differed between time points. While none of these factors are avoidable, they introduce additional variability into our sample and prevent an accurate direct statistical comparison between years. Finally, the results from a single institution may not be generalizable; however, our sample included $61 \%$ of all neurological surgery applicants in 2012..$^{17}$

To certify their application, all applicants must acknowledge an honor statement which states: "I understand that any false or missing information may disqualify me from consideration for a position; may result in an investigation by the AAMC [Association of American Medical Colleges] ...or if employed, may constitute cause for termination." Unfortunately, we have learned that the honor system previously applied in this realm is not sufficient. These results indicate that the applicants with greater numbers of total reported works on their $\mathrm{CV}$ and those from unranked US medical schools were more likely to have erroneous citations. However, given the lack of specificity of these findings, it is advisable that programs employ increased vigilance when reviewing all applications.

This study highlights an alarming trend among young recruits to neurosurgery and leads to concerns that this may also be a problem in their mentors at higher levels of the field. These results are an important reminder that maintaining impeccable research ethics is the responsibility of all medical leaders, and research advisors must lead by example. We advocate improved education and supervision of medical students so that they are not misinformed regarding the critical nature of accurate reporting or its consequences.

\section{Conclusions}

Misrepresentation of scholarly achievements is an in- 
creasing problem among neurosurgery residency applicants. We believe immediate widespread attention to this issue is necessary to prevent perpetuation of a belief that such behavior is acceptable.

\section{References}

1. Beswick DM, Man LX, Johnston BA, Johnson JT, Schaitkin BM: Publication misrepresentation among otolaryngology residency applicants. Otolaryngol Head Neck Surg 143:815-819, 2010

2. Boyd AS, Hook M, King LE Jr: An evaluation of the accuracy of residency applicants' curricula vitae: are the claims of publications erroneous? J Am Acad Dermatol 35:606-608, 1996

3. Caplan JP, Borus JF, Chang G, Greenberg WE: Poor intentions or poor attention: misrepresentation by applicants to psychiatry residency. Acad Psychiatry 32:225-229, 2008

4. Cohen-Gadol AA, Koch CA, Raffel C, Spinner RJ: Confirmation of research publications reported by neurological surgery residency applicants. Surg Neurol 60:280-284, 2003

5. Dale JA, Schmitt CM, Crosby LA: Misrepresentation of research criteria by orthopaedic residency applicants. J Bone Joint Surg Am 81:1679-1681, 1999

6. Engler RL, Covell JW, Friedman PJ, Kitcher PS, Peters RM: Misrepresentation and responsibility in medical research. $\mathbf{N}$ Engl J Med 317:1383-1389, 1987

7. Gasior AC, Knott EM, Fike FB, Moratello VE, St Peter SD, Ostlie DJ, et al: Ghost publications in the pediatric surgery match. J Surg Res 184:37-41, 2013

8. Girgis F: Feasibility of a dual neurosurgeon-scientist career in Canada: a survey study. Can J Neurol Sci 40:504-511, 2013

9. Goe LC, Herrera AM, Mower WR: Misrepresentation of research citations among medical school faculty applicants. Acad Med 73:1183-1186, 1998

10. Gurudevan SV, Mower WR: Misrepresentation of research publications among emergency medicine residency applicants. Ann Emerg Med 27:327-330, 1996

11. Hebert RS, Smith CG, Wright SM: Minimal prevalence of authorship misrepresentation among internal medicine residency applicants: do previous estimates of "misrepresentation" represent insufficient case finding? Ann Intern Med 138:390-392, 2003

12. Hsi RS, Hotaling JM, Moore TN, Joyner BD: Publication misrepresentation among urology residency applicants. World J Urol 31:697-702, 2013

13. Kimball HR: Credentials misrepresentation: another challenge to professionalism. Ann Intern Med 123:58-59, 1995

14. Konstantakos EK, Laughlin RT, Markert RJ, Crosby LA:
Follow-up on misrepresentation of research activity by orthopaedic residency applicants: has anything changed? J Bone Joint Surg Am 89:2084-2088, 2007

15. Man LX, Beswick DM, Johnson JT, Schaitkin BM: Postmatch findings in otolaryngology residency applicants with publication misrepresentation. Otolaryngol Head Neck Surg 144:1011-1012, 2011

16. Maverakis E, Li CS, Alikhan A, Lin TC, Idriss N, Armstrong AW: The effect of academic "misrepresentation" on residency match outcomes. Dermatol Online J 18:1, 2012

17. National Resident Matching Program: Results and Data: 2013 Main Residency Match. Washington DC: National Resident Matching Program, 2013, p 116

18. Neuman SA, Long TR, Rose SH: Publication misrepresentation among anesthesiology residency applicants. Anesth Analg 112:674-677, 2011

19. Patel MV, Pradhan BB, Meals RA: Misrepresentation of research publications among orthopedic surgery fellowship applicants: a comparison with documented misrepresentations in other fields. Spine (Phila Pa 1976) 28:631-636, 2003

20. Phillips JP, Sugg KB, Murphy MA, Kasten SJ: Misrepresentation of scholarly works by integrated plastic surgery applicants. Plast Reconstr Surg 130:731-735, 2012

21. Sekas G, Hutson WR: Misrepresentation of academic accomplishments by applicants for gastroenterology fellowships. Ann Intern Med 123:38-41, 1995

22. Wiggins MN: A meta-analysis of studies of publication misrepresentation by applicants to residency and fellowship programs. Acad Med 85:1470-1474, 2010

23. Wiggins MN: Misrepresentation by ophthalmology residency applicants. Arch Ophthalmol 128:906-910, 2010

\section{Author Contributions}

Conception and design: Kistka, Nayeri, Dow, Chambless. Acquisition of data: Nayeri, Dow. Analysis and interpretation of data: Kistka, Nayeri, Wang, Chandrasekhar, Chambless. Drafting the article: Kistka. Critically revising the article: Kistka, Nayeri, Dow, Chandrasekhar, Chambless. Reviewed submitted version of manuscript: all authors. Approved the final version of the manuscript on behalf of all authors: Kistka. Statistical analysis: Kistka, Nayeri, Wang, Chandrasekhar. Administrative/technical/material support: Dow, Chambless. Study supervision: Chambless.

\section{Correspondence}

Heather M. Kistka, Department of Neurosurgery, Vanderbilt University Medical Center, 1161 21st Ave. S, Rm. T-4224 MCN, Nashville, TN 37232-2380. email: heather.kistka@vanderbilt.edu. 